\title{
POLLURISK: AN INNOVATIVE EXPERIMENTAL PLATFORM TO INVESTIGATE HEALTH IMPACTS OF AIR QUALITY
}

\author{
PATRICE COLL ${ }^{1}$, MATHIEU CAZAUNAU ${ }^{1}$, JORGE BOCZKOWSKI ${ }^{2}$, MAËVA ZYSMAN ${ }^{2}$, \\ JEAN-FRANÇOIS DOUSSIN ${ }^{1}$, ALINE GRATIEN ${ }^{1}$, GENEVIÈVE DERUMEAUX ${ }^{2}$, MARIA PINI $^{2}$, \\ CLAUDIA DI BIAGIO ${ }^{1}$, ÉDOUARD PANGUI ${ }^{1}$, CÉCILE GAIMOZ $^{1}$, SOPHIE HÜE ${ }^{2}$, FRÉDÉRIC RELAIX ${ }^{2}$, \\ AUDREY DER VATANIAN ${ }^{2}$, ISABELLE COLL ${ }^{1}$, VINCENT MICHOUD ${ }^{1}$, PAOLA FORMENTI ${ }^{1}$, \\ GILLES FORÊT ${ }^{1}$, LAURENCE THAVARATNASINGAM ${ }^{1}$, ADELA AMAR $^{1}$, \\ MICKAËL LACAVALERIE ${ }^{2}$, MARGAUX MÄDER ${ }^{1} \&$ SOPHIE LANONE ${ }^{2}$ \\ ${ }^{1}$ LISA UMR CNRS 7583, Universities Paris Est Créteil and Paris Diderot, France \\ ${ }^{2}$ IMRB - Inserm U955, Faculté de Médecine de Créteil, France
}

\begin{abstract}
The World Health Organization (WHO) estimated that there were 3.7 million premature deaths due to air pollution in 2014, confirming that air pollution is a great environmental risk to health. Air pollution is responsible for a loss of more than $3 \%$ of productivity (via premature death, incapacity for work due to diseases, etc.). The studies conducted so far show that the effects of air pollution on health depend not only on the quality of the surrounding air, but also on the subjects exposed and their individual vulnerability (asthma, COPD, obesity, etc.). Despite the evidence on the adverse health effects of exposure to air micro-pollutants there are still uncertainties about the nature of these effects and progress to be made on their quantification. This limitation of our knowledge is mainly attributed to the complexity of the polluted atmospheres, and to the great difficulty to model the impact of realistic situations of exposure. Among the constituents of air pollution individually associated with deleterious effects on health, we consider gaseous pollutants $\left(\mathrm{O}_{3}, \mathrm{SO}_{2}, \mathrm{CO}, \mathrm{NO}_{x}, \mathrm{VOC} \ldots\right)$ and particles $\left(\mathrm{PM}_{10}\right.$, $\mathrm{PM}_{2.5}$, ultrafine). In order to realistically simulate atmospheric mixtures in all their complexity in the laboratory, environmental chemists have developed photo-reactors that are equipped to reproduce and control atmospheric processes such as solar radiation, concentrations of species and the timely injection of aerosols and gases. These atmospheric simulation chambers thus offer the possibility of studying the myriad of products resulting from the atmospheric oxidation of primary compounds. Using CESAM, an atmospheric simulation chamber (cesam.cnrs.fr), we have developed a totally innovative platform for exposing mice to realistic atmospheric conditions. Here we present the first toxicological analyses of the organs of these mice after 48 hours of exposure, carried out as part of feasibility experiments aimed at testing this experimental concept, as well as other preliminary results.

Keywords: air quality, health impacts, atmospheric chemistry, pollutants, aerosols, SOA, chronic obstructive pulmonary disease, asthma.
\end{abstract}

\section{INTRODUCTION}

One of the first alerts of the major effects of air pollution on health took place during an episode in London during the winter of 1952: during 4 days, a thick fog fell on the city, and 4000 additional deaths compared to the same period in previous years were recorded, more than half of which were for respiratory reasons [1]. The World Health Organization (WHO) has recently estimated that in 2012 alone, 3.7 million deaths occurred prematurely because of air pollution (www.who.int). In 2014 they declared the pollution atmospheric as representing the greatest environmental risk to health and responsible for a loss of more than $3 \%$ of productivity due to premature deaths and incapacity for work. According to a 2012 report from the Regional Health Observatory in Ile de France (Paris region) for the period 2004-2006, air pollution has been responsible, at least annually, for: 
- In the short term, about one hundred expected deaths and about 480 hospitalizations for cardiorespiratory causes, which represents a total cost of about 11.4 million euros per year.

- In the long-term (chronic exposure), about 1400 expected adult deaths, or $4 \%$ of all non-accidental registered deaths, which results in an average loss of 6 months of life expectancy at 30 years per person. This impact represents a cost of around 2.3 billion euros per year.

Numerous epidemiological studies, published in the literature after controlled human exposure, show that acute exposure to peaks of atmospheric pollution is accompanied, at the respiratory level, by a decrease in respiratory function in healthy adult patients, exaggerated morbidity and mortality in patients with underlying lung diseases such as asthma or chronic obstructive pulmonary disease (COPD), or patients with airway hyper-responsiveness or obese. Chronic exposure to high pollution is associated with decreased respiratory function in adults, as well as the development of cardiovascular pathologies and lung cancers. In addition, deleterious effects on lung growth and respiratory function have been reported in children exposed to high levels of air pollution. Cardiorespiratory and neurological effects have also been reported, with air pollution contributing to approximately $34 \%$ of stroke deaths and $27 \%$ of those due to heart disease (www.who.int).

From all these studies, it is important to note that the effects of air pollution on health depend not only on air quality, but also on the subjects concerned and their individual vulnerability. Patients with a morbid state (asthma, COPD, obesity), or at certain ages of life (children, elderly patients) have a particular susceptibility to pollution. Recent studies suggest that exposure to pollution during the intrauterine period could have deleterious consequences on foetal growth and development (decreased birth weight, increased risk of premature birth, ...). In addition, there are described effects concerning more specifically the respiratory health of children exposed in utero, such as a positive association with the number of days with wheezing episodes at 2 years of age, with the prevalence of asthma or decreased respiratory function in adolescence. A recent study shows that exposure of pregnant females to nanoparticles of cerium dioxide (which can be found in diesel combustion, for example) causes respiratory problems in their offspring, which continues into adulthood [2]. This result is all the more important as it could represent a susceptibility factor for developing pathologies in adulthood.

Despite the evidence pointing towards the health effects of maternal exposures to air pollution there are still uncertainties due to the complexity of the atmospheres studied, which are difficult to characterize, quantify and therefore model in real conditions of exposure. One of the main challenges in the context of the study of the health effects of air pollution results from the extreme complexity of the atmospheric mixture: at any point in space, thousands of pollutants with important chemical diversity coexist at concentrations ranging from ppm to ppt, producing an instantaneous chemical reactivity that cannot be estimated with conventional methods of organic chemistry. In addition, this reactivity is highly modulated over time due to the rapid transformation of many species on time scales from the second to the minute. Air pollution thus consists of a complex mixture of several thousand components that can be either primary (generated mainly from the combustion of fossil energy by motor vehicles or industrial processes, by domestic or commercial activities, ...), or secondary (formed from the reaction of primary pollutants with sunlight, oxides of nitrogen, oxygen, or other oxidants contained and / or produced in the atmosphere). 


\section{CHALLENGES}

It is now recognized that it is necessary to understand our environment in order to be able to isolate its causes of various human pathologies. In this context, the environmental related pathologies are those not associated with genetic or behavioural (e.g. eating or smoking) dispositions or food contamination [3].

On the basis of this definition, chronic pathologies linked to the environment range from hormone-dependent and non-hormone-dependent cancers to developmental diseases, including impairment of fertility, neurological diseases, obesity, cardiological diseases and respiratory diseases, autoimmune diseases, and allergies for example.

Approaches to conducting studies in this area of the toxicological impact of the environment are diverse (i.e. by organ, pathology, source, substance, mechanism, etc). These xenobiotic studies are subdivided into 3 main axes, namely:

- Toxicity by chemical reactivity or direct physical interference (the most "classical" route)

- Stress adaptation toxicity (metabolic reactions)

- The toxicological impact of homeostasis (endocrinology)

From the point of view of health, the constituents of air pollution individually identified as being associated with deleterious effects are gaseous pollutants (ozone $-\mathrm{O}_{3}$, sulfur dioxide - $\mathrm{SO}_{2}$, carbon monoxide $-\mathrm{CO}$, nitrogen oxides $-\mathrm{NO}_{\mathrm{x}}$, volatile organic compounds $-\mathrm{VOCs}$, including benzene, etc.) and particulate matter (PM). We distinguish mainly 3 classes of PM concentrations, categorized according to their size: coarse ( $\mathrm{PM}_{10}$, diameter between 2.5 and 10 microns), fine ( $\mathrm{PM}_{2.5}$, diameter between 0.1 and 2.5 microns) and finally ultrafine ( $\mathrm{PM}_{0.1}$, with a diameter of less than $0.1 \mu \mathrm{m})$. However, the relevance of an approach based on the study of components isolated from pollution is undermined by the lack of consideration of the suspected synergy between the different constituents of air pollution. Another approach to studying the effects of air pollution on health is to expose living organisms to outdoor air under real conditions. The main defect of this approach is the diversity of pollution rates to which organisms may be exposed, due to the high temporal variability of emission, meteorology and atmospheric dispersion, as well as the difficulty in finely controlling reproducible experimental conditions. A complementary approach is to propose "exposomes", i.e. to realistically simulate, in the laboratory, an atmospheric mixture in all its complexity. In this context, and in order to study the myriad of products resulting from the atmospheric oxidation of primary organic compounds, environmental chemists have developed atmospheric simulation chambers. These chambers are equipped with photoreactors, with protocols that mimic atmospheric conditions such solar radiation, realistic concentrations, the injection primary aerosols and the formation of secondary aerosols.

The LISA (Interuniversity Laboratory for Atmospheric Systems, UMR CNRS 7583) has been developing such equipment for more than twenty years and has become a worldrenowned player in experimental atmospheric simulation (for example, LISA coordinates the European infrastructure network of atmospheric simulation chambers, EuroChamp 2020 (www.eurochamp.org)). In the early 2010s, LISA in particular completed the development of the CESAM chamber (http://cesam.cnrs.fr), which now has the status of National Instrument INSU/CNRS, to study multiphase atmospheric chemistry by controlled generation of particulate pollution mixtures. It is this reactor with its unique performance that we use as part of our PolluRisk project (see the description of CESAM on the referenced website). 


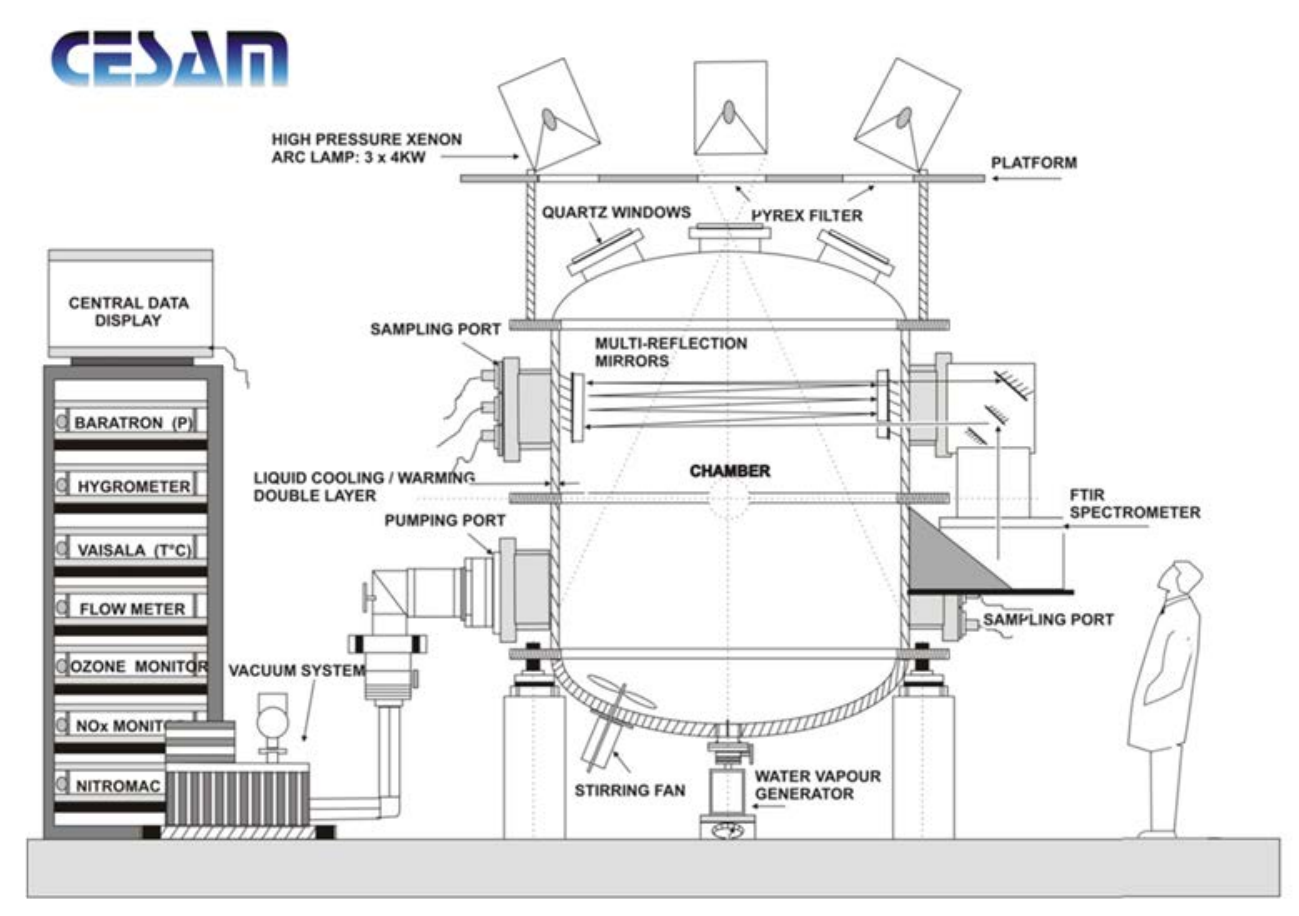

Figure 1: A schematic view of the CESAM chamber.

\section{INNOVATIVE PROTOCOL}

The innovative approach we set up to support such studies is to realistically simulate, at the laboratory, the atmospheric mixture in all its complexity, thus keeping the ability to control, reproduce and carefully characterize the experimental conditions. We used the CESAM chamber $\left(4.2 \mathrm{~m}^{3}\right.$ stainless steel atmospheric simulation, evacuable down to a few $10^{-7} \mathrm{~atm}$, temperature controlled between $+15^{\circ} \mathrm{C}$ and $+60^{\circ} \mathrm{C}$ ) in order to study the myriad of products arising from the atmospheric oxidation of primary organic compounds.

The experimental protocol consists in the continuous injection of relevant mixtures of primary pollutants (mainly nitrogen oxides, organic compounds from a representative mix of anthropogenic emissions, sulphur dioxide, soot, inorganic salts and potentially mineral dust particles if needed - e.g. to simulate Beijing's atmosphere) at low concentrations (ppb levels) in air in the CESAM simulation chamber operated as a slow flow reactor. The residence time of simulated air parcels in the experimental volume is fixed to 4 hours, in order to represent air masses of regional scale. During this time the synthetic mixture is exposed to an artificial solar irradiation, allowing secondary pollutants such as ozone, nitric acid, formaldehyde, peroxyacetyl nitrate (PANs) as well as complex polyfunctional organics including SOA (Secondary Organic Aerosol) to be produced and to reach their chemical steady state. Living organisms are exposed to constant flows of such a mixture during time scales of week to test their effect on living organisms.

In order to scale the pollutant concentrations in the initial mix, we were able to run a predictive model to estimate the concentrations of all gaseous and particulate species in CESAM, after a residence time of 4 hours in the chamber. Below is an illustration of such a modelling in the case of an atmosphere relevant to the Paris area (Fig. 2). 


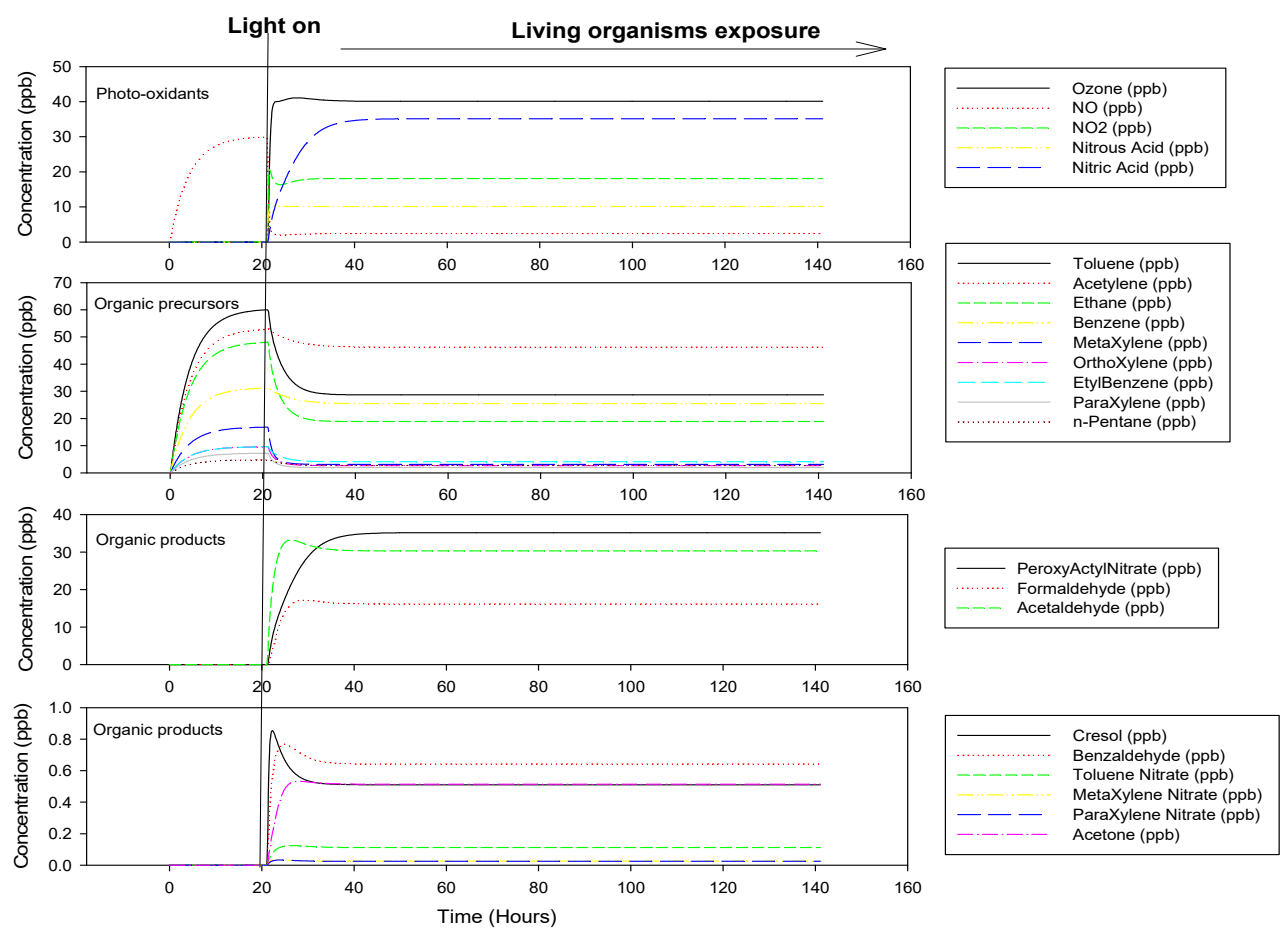

Figure 2: Numerical modelling of the chemical activity inside CESAM chamber, describing the photo-stationary concentrations for the photoproducts (as cresol or acetone plot on bottom graph), 20 hours later than the injection of 9 precursors.

The model takes into account the injection of 9 precursors (gaseous chemicals) in the simulation chamber: nitrous acid, toluene, acetylene, ethane, benzene, meta-xylene, orthoxylene, para-xylene, ethyl-benzene and n-pentane. After 20 hours of flowing and mixing in the chamber (this duration can be reduced to some hours by increasing the concentrations and flows of precursors) the targeted concentrations of pollutants are obtained (here in reference to what was measured on the field by [4]). Then the light simulating the sunlight irradiation is switched on and taking into account in the order of 1500 reactions involving around 3000 chemicals the model predict what will be the photo-stationary concentrations in the chamber for any of those photoproducts. We then plot some of them as an illustration, as cresol or acetone (bottom graph).

Once the requested steady-state CESAM experimental conditions are reached then a desired volumetric flowrate is transferred to the compartment where the living organisms are exposed, due to a pressure of 11 millibars inside CESAM. In each experiment, the compartments undergo a constant air flow from the simulation chamber. Furthermore, we make sure the path between the chamber and the exposure devices is minimized, in order to preserve the numerous reactive chemicals, present in the simulated atmosphere.

The CESAM laboratory is equipped with a comprehensive set of analytical instruments and benefit from the instrumental environment dedicated to atmospheric chemistry provided 
by CNRS-LISA (http://www.cesam.cnrs.fr), especially gaseous pollutants analysers $\left(\mathrm{O}_{3}\right.$, $\mathrm{NO}, \mathrm{NO}_{2}, \mathrm{SO}_{2}, \mathrm{CO}, \mathrm{CO}_{2}, \mathrm{COV} \ldots$ ) and aerosols properties (mass and size distributions, hygroscopicity...). This allows us to accurately and precisely measure the time evolution of the pollutants concentrations within the different exposure compartments, as shown in the Fig. 3 for aerosol particles.

There are three different ways to introduce solid particles in the CESAM chamber:

a) inorganic aerosols : inorganic particles are continuously injected in CESAM from a salt(s) solution (e.g. $\left[\mathrm{NH}_{4}\right]_{2} \mathrm{SO}_{4}$ solution in the $10^{-4} / 10^{-3} \mathrm{Mol} .1^{-1}$ ) using a commercial atomizer (TSI 3076 atomizer), with a transfer rate to CESAM relevant to the targeted final concentration, as described in [5].

b) Soot introduction: for the control of continuous soot introduction in CESAM, a second chamber is used as a "soot reservoir". This chamber will be named "CAC" ("CESAM Auxiliary Chamber") in the rest of the text. CAC has a volume of $2 \mathrm{~m}^{3}$ and is equipped with a pumping system, a fan and a 0-1000 Torr pressure sensor. It is directly connected to CESAM by a 1/4" Stainless Steel tube. During the introduction of soot into the tank enclosure, the two chambers are isolated from each other. The use of a miniCAST (model 5201A) allows us to fill the tank enclosure with a known mass of soot. This quantity is determined according to the final quantity desired in CESAM. When the introduction is complete, the two chambers are connected and a fixed nitrogen flow allows the introduction of soot continuously from the CAC to CESAM. The frequency of introduction of soot by the CAST is determined according to the dilution rate in CESAM. A comparison of the model used to determine the introductory parameters and the measurements performed in CESAM is displayed in Fig. 4. The difference between the model and the experiment comes from the aging of the aerosol (coagulation + loss) in CAC, which are not taken into account in the model.

The experimental parameters used are the following:

Concentration in CAC : $\quad 4000 \mu \mathrm{g} / \mathrm{m}^{3}$

Transfer rate CESAM/CAC : $\quad 0.5 \mathrm{l} / \mathrm{min}$

Dilution rate in CESAM : $\quad 15 \mathrm{l} / \mathrm{min}$

Frequency of introduction of soot in CAC : 1 daily

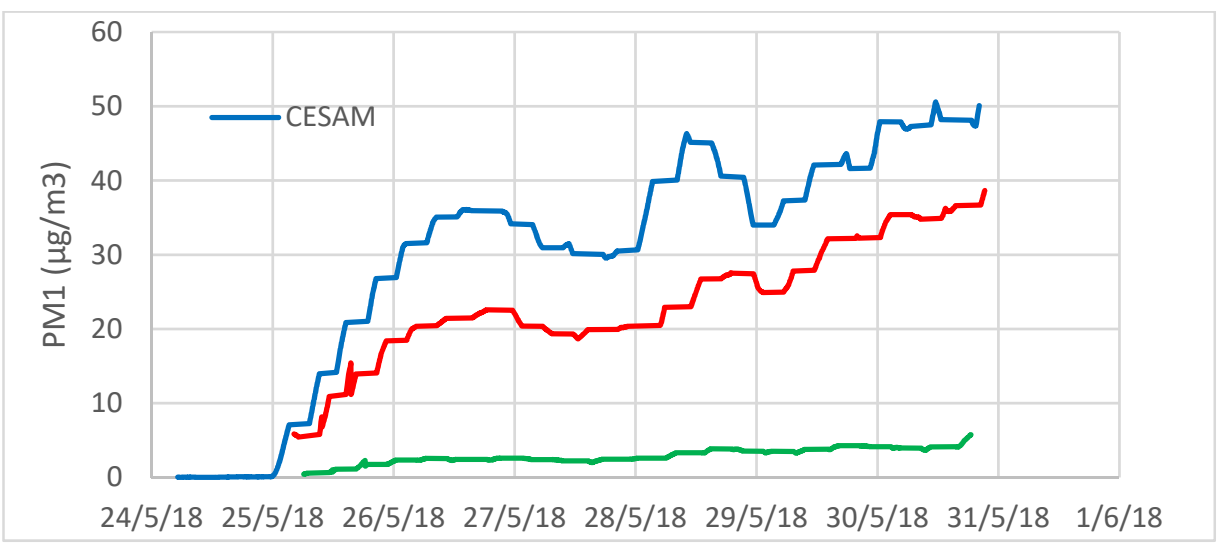

Figure 3: Evolution of the total particles concentration as a function of time, successively measured in the different compartments: in blue in CESAM chamber, in red in the compartment where living organisms are exposed, in green in the "reference" compartment. 


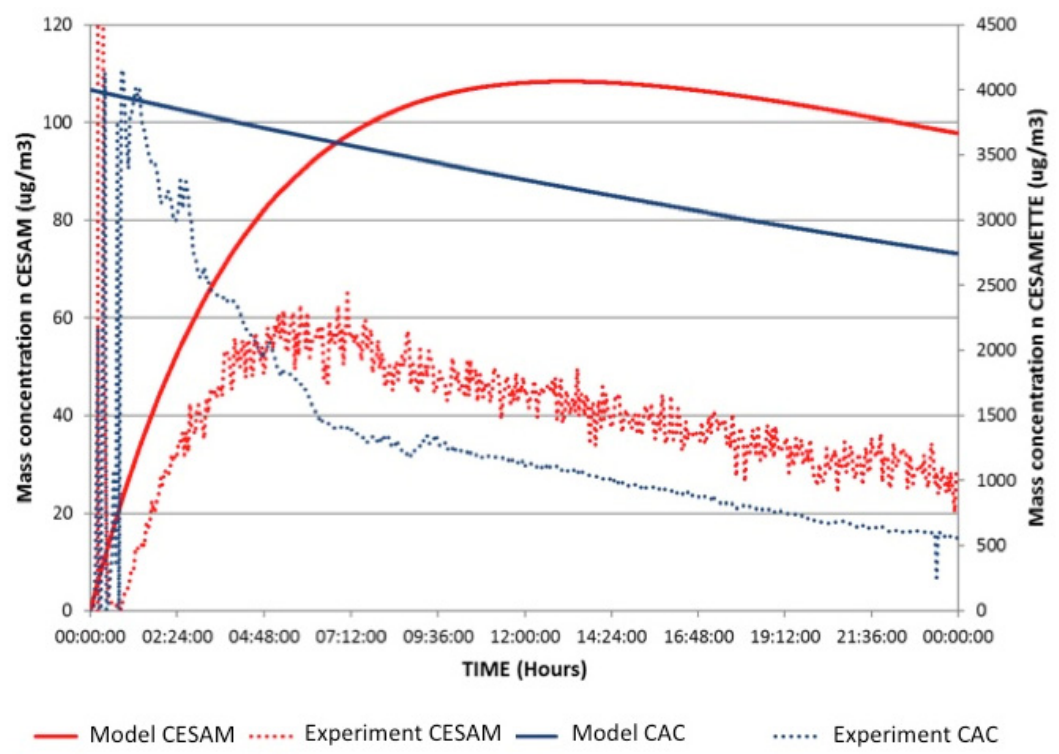

Figure 4: Comparison between the model used to determine the initial parameters and the measurements performed in CESAM.

c) dust particles: about $15 \mathrm{~g}$ of soil sample (Gobi Desert soil in the case of Beijing's simulation) is placed in a Büchner flask and shaken for about $30 \mathrm{~min}$ at $100 \mathrm{~Hz}$ by means of a sieve shaker (Retsch AS200). The dust suspension in the flask is then injected into the chamber by flushing it with $\mathrm{N}_{2}$ at $10 \mathrm{~L} \mathrm{~min}^{-1}$ for about $10-15 \mathrm{~min}$, whilst continuously shaking the soil. This injection is done as needed (for the example of our Beijing's simulation it is once per day, as a simulation of a daily dust storm arising Beijing) [6].

\section{PRELIMINARY INVESTIGATIONS}

Taking benefit of this PolluRisk platform, we ran an experiment (reproduced 2 times) where murine models have been exposed to a Paris simulated atmosphere for $48 \mathrm{~h}$. Mice were separated in 2 different batches. One was exposed in a dedicated compartment to the atmosphere directly arriving from CESAM (see section 3), and the other exposed in another dedicated compartment to a "reference atmosphere" (Atmosphere from CESAM passing through several filters removing all micro-pollutants, both in gaseous and atmospheric phase). In each compartment, animals were installed in exposure cages ( 5 adults maximum per cage), provided by @Techniplast. These cages contain clean litter, and the animals were supplied with water (baby bottles) and food. The exposure time was 2 days, which did not require the change of animals between the beginning and the end of the experiment. The temperature and hygrometry inside the exposure chamber were monitored continuously (in the range $[30 \%-50 \%]$ for the relative humidity and in the range $\left[21^{\circ} \mathrm{C}-26^{\circ} \mathrm{C}\right]$ for the temperature). On the other hand, a day/night cycle of 12 hours was simulated with lights located inside the exposition enclosure. An orifice in the door of the exposure chamber makes it possible to check the activity of a cage (which serves as an "activity indicator" for all the stands) continuously. Throughout the exposition, the animals were monitored by means of cameras present inside the exhibition hall. If an anomaly was observed (lack of movement of 
the mice, insufficient food or water, signs of distress etc), the enclosure was opened and action taken to correct the anomaly.

Ethics: The project (file number 17-087, $n^{\circ}$ APAFIS 2017113013439718) received a favourable opinion from the ComEth on December 12, 2017. The project is valid for 5 years.

\section{PRELIMINARY RESULTS}

Fig. 6 and Fig. 7 summarize part of the results that will be presented more extensively during the Air Pollution conference. They mainly concern toxicological analyses of organs or tissues sampled on mice after their exposure.

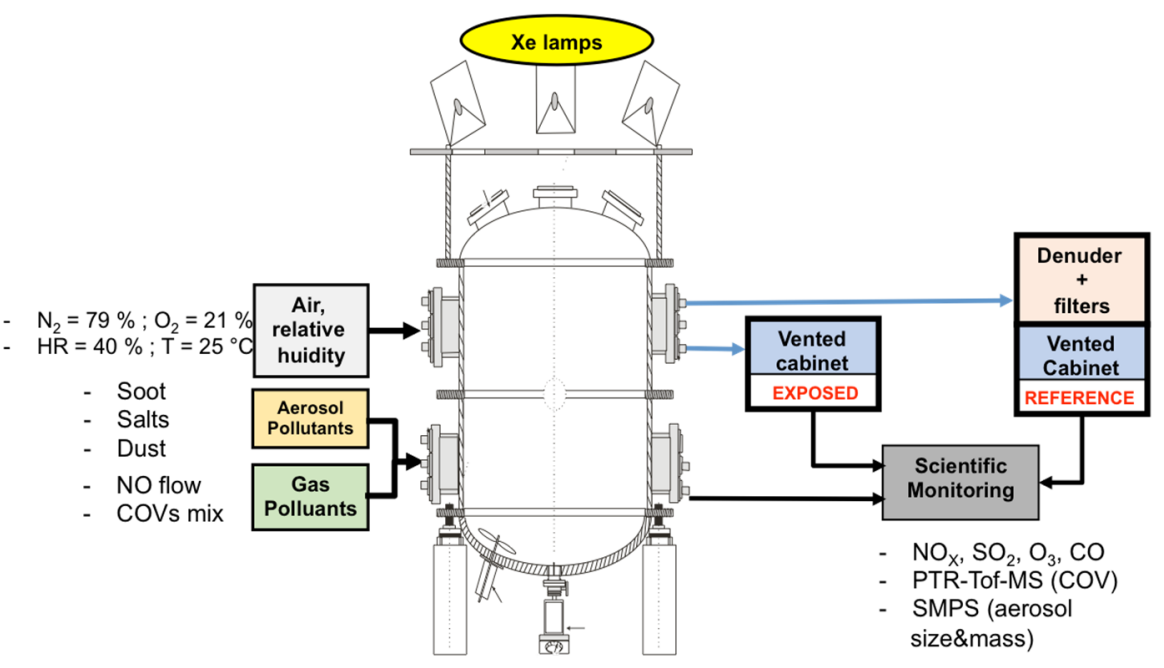

Figure 5: A schematic view of the PolluRisk protocol.
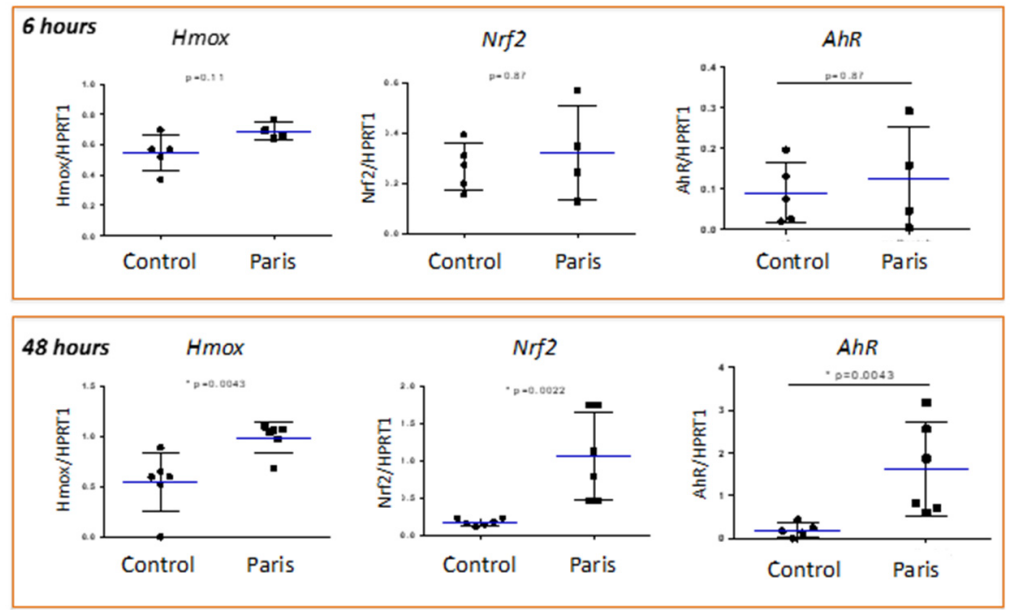

Figure 6: In the lung, few modifications were observable after 6 hours of exposure, but a typical response attesting the presence of oxidative stress (increased expression of Hmox and Nrf2) and xenobiotics (Ahr) was detected at $48 \mathrm{~h}$. 

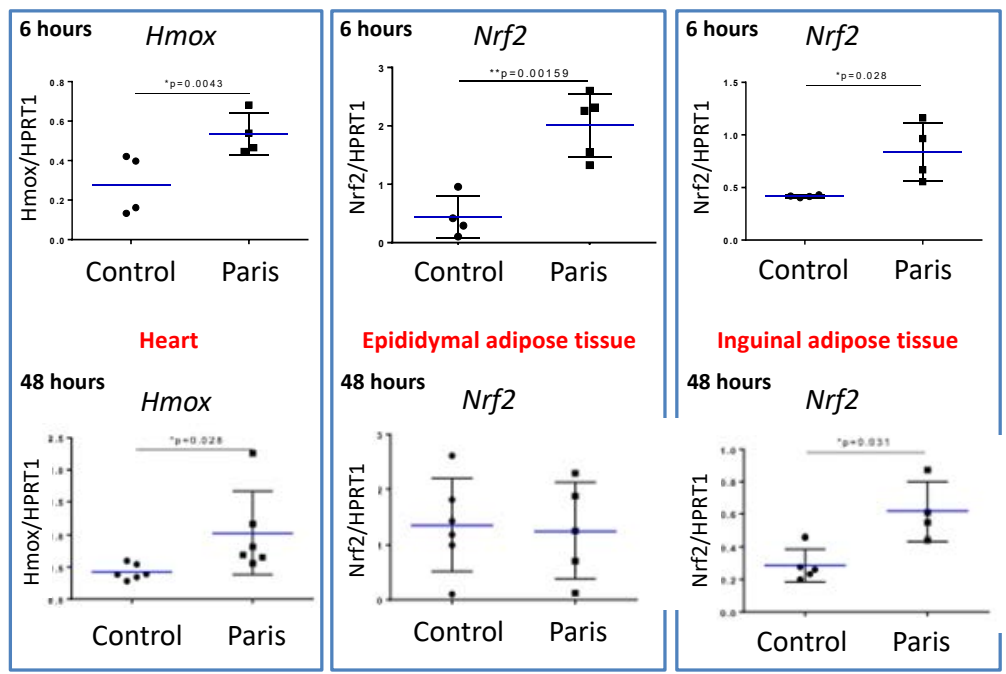

Figure 7: The heart and adipose tissue are affected earlier than the lung.

\section{ACKNOWLEDGEMENTS}

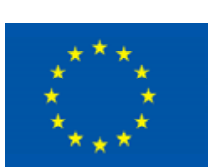

This project/work has received funding from the European Union's Horizon 2020 research and innovation programme through the EUROCHAMP-2020 Infrastructure Activity under grant agreement $\mathrm{N}^{\circ}$ 730997. We also thank CNRS/INSU, INSERM, Région Ile de France and UPEC. In addition, we deeply thank Marc Mallet (LISA) for English revision.

\section{REFERENCES}

[1] Logan, W.P.D. et al., Mortality in the London fog incident, 1952. Lancet, 261(6755), pp. 336-338, 1953.

[2] Paul, E. et al., Pulmonary exposure to metallic nanomaterials during pregnancy irreversibly impairs lung development of the offspring. Nanotoxicology, 11(4), pp. 484495, 2017.

[3] Lim, S.S. et al., A comparative risk assessment of burden of disease and injury attributable to 67 risk factors and risk factor clusters in 21 regions, 1990-2010: a systematic analysis for the Global Burden of Disease Study 2010. Lancet, 380(9859), pp. 2224-2260, 2012.

[4] Monod, A., Sive, B.C., Avino, P., Chen, T., Blake, D.R. \& Rowland, S., Monoaromatic compounds in ambient air of various cities: a focus on correlations between the xylenes and ethylbenzene. Atmospheric Environment, 35, pp. 135-149, 2001.

[5] Wang, J. et al., Design of a new multi-phase experimental simulation chamber for atmospheric photosmog, Aerosol and cloud chemistry research. Atmospheric Measurement Techniques, 4, pp. 2465-2494, 2011.

[6] Di Biagio, C., et al., Global scale variability of the mineral dust long-wave refractive index: a new dataset of in situ measurements for climate modeling and remote sensing. Atmos. Chem. Phys., 17, pp. 1901-1929, 2017. 